\title{
Enhancement of the Transition to Detonation of a Turbulent Hydrogen-Air Flame by Nanosecond Repetitively Pulsed Plasma Discharges
}

\author{
Joshua A.T. Gray*, Deanna A. Lacoste \\ King Abdullah University of Science and Technology (KAUST), Clean Combustion \\ Research Center, Thuwal 23955-6900, Saudi Arabia
}

\begin{abstract}
This work provides proof of concept for the use of nanosecond repetitively pulsed (NRP) plasma discharges to accelerate a propagating turbulent flame, resulting in enhanced deflagration-to-detonation transition and significant reduction in run-up length. The investigations are conducted on a stoichiometric hydrogen-air mixture at near ambient conditions. The effect of plasma actuation on the flame velocity is investigated using time-of-flight measurements of the propagating flame and detonation wave. The flame velocity shortly after the application of the NRP plasma discharges is more than double that obtained in cases in which no plasma is applied. High-speed imaging of $\mathrm{OH}^{*}$ chemiluminescence in the electrode area confirms this result and provides insight about the mechanisms of plasma action. While the volumetric energy deposited during plasma actuation is sufficiently low as to not ignite the combustible mixture prior the arrival of the flame, the chemical and thermal enhancement of the gas is efficient enough to significantly
\end{abstract}

${ }^{*}$ Corresponding author.

Email address: joshua.gray@kaust.edu.sa (J.A.T. Gray) 
accelerate the transition to detonation. The decrease in the run-up length to transition to detonation is obtained for a plasma power of less than $0.14 \%$ of the thermal power of the flame. This result indicates that low-energy active devices using NRP discharges might be suitable for replacing passive devices such as orifice plates or Shchelkin spirals.

Keywords: deflagration-to-detonation transition, plasma-assisted combustion, non-equilibrium plasma, flame acceleration

\section{Introduction}

The need for more efficient cycles for combustion-based energy production has led to much attention being paid to pressure-gain combustion cycles in recent years (e.g., [1-6]). These cycles are inherently more efficient and will allow not only for an easier transition to but also, when utilizing hydrogen as a fuel, an efficient coupling with renewable energy sources in the future [7-9]. One such pressure-gain cycle is known as the Fickett-Jacobs cycle [10], which is based on the reaction of fuel and oxidizer by means of a detonation wave. The so-called pulse detonation combustor (PDC) is one concept that utilizes this thermodynamic cycle.

Due to energetic considerations, however, the detonation is typically not directly initiated, but rather the gaseous mixture is ignited by means of a lowenergy source and the resulting flame is accelerated until the phenomenon known as the deflagration-to-detonation transition (DDT) occurs [11]. Frequently, obstacles are used in order to induce DDT (e.g., [12-15]). However, both the number of these obstacles as well as the run-up length to the occurrence of DDT have a direct impact on the efficiency of the PDC [16]. 
Furthermore, the obstacles may be subjected to extreme thermal loading and subsequent failure. For these reasons, it may be argued that limiting the number of obstacles or eliminating them altogether for the purpose of inducing DDT is an enabling step for pulse detonation technologies.

An alternative solution is the use of electrical discharges to decrease the run-up length to DDT. Arc discharges have been successfully used to enhance DDT $[17,18]$, but the electrical energy deposition of these thermal plasmas is too high to consider them for an actuator that can be efficiently used in a PDC. For example, by using a combination of several discharges, Frolov et al. [18] successfully reduced the electrical energy deposition necessary for the onset of a detonation down to $100 \mathrm{~J}$, which remains a few orders of magnitude higher than a conventional ignition system for gas turbines or car engines. In addition, the synchronization of such distributed arc discharges, each of them acting as a local ignition source, is extremely important and challenging. A non-optimized synchronization could induce adverse effects on the DDT [19].

On the other hand, non-equilibrium plasma produced by nanosecond repetitively pulsed (NRP) discharges has shown promising results in plasmaassisted combustion [20, 21]. These NRP discharges have aroused a strong interest from the scientific community because they are extremely efficient in producing, under conditions relevant for combustion applications, highly reactive mixtures at low energetic cost [22-25]. For detonation applications, NRP discharges have been successfully used as a more efficient ignition source than conventional methods [26-31]. For example, Busby et al. [27] have shown in a PDC filled with stoichiometric gasoline-air mixture that ignition by nanosecond pulsed discharges transverse to the flow can reduce both the 
ignition delay time and the DDT by up to $45 \%$ compared to a conventional ignition system. In Starikovskiy et al. [30], DDT in propane-oxygen-enriched air mixtures was achieved in less than $0.5 \mathrm{~ms}$ by using a nanosecond ignition system with an ignition energy as low as $300 \mathrm{~mJ}$. In all of these studies, even if the plasma sources are distributed over several centimeters, the phenomenon of flame ignition and acceleration are coupled. Of course, this coupling is a good strategy for detonation engines in which decreasing both the ignition energy and the time (or length) of transition to detonation are of primary importance for the efficiency. However, in these cases, the understanding and optimization of the effect of non-equilibrium plasma on the acceleration of turbulent flames is difficult to achieve.

To the best of our knowledge, the acceleration of an existing turbulent flame up to its transition to a detonation by plasma discharges with energy deposition below the minimum ignition energy (MIE) has never been demonstrated. In this paper, the authors propose a novel approach of using nanosecond repetitively pulsed (NRP) plasma discharges not for ignition enhancement, but rather to accelerate a turbulent propagating flame and in this way, decrease the run-up length to DDT. The objective is to assess if low-energy active devices using NRP discharges could be considered as an alternative to passive flame accelerators such as orifice plates or Shchelkin spirals.

\section{Experimental setup and procedure}

A detailed schematic of the test apparatus, including locations of sensors, pumps, gas lines, and the electrode configuration is shown in Fig. 1. 
The experiments are conducted in a closed stainless steel tube with an inner diameter of $39 \mathrm{~mm}$ and a length of $3350 \mathrm{~mm}$. One of two commercial spark plugs (NGK CR8E 1275 or Enker TE70) is installed at the center of the head wall at the ignition end, depending on the desired built-in resistance. A single orifice plate with a blockage ratio of 0.43 is located at a distance of $540 \mathrm{~mm}$ from the head wall. A transparent acrylic glass tube with a length $100 \mathrm{~mm}$ is installed at a distance of $1080 \mathrm{~mm}$ from the head wall. The electrodes for the NRP discharges are installed in the acrylic glass tube, which serves to electrically insulate the electrodes from the test bench. Downstream of the electrodes are four tube sections, each containing two piezoelectric pressure transducers (PCB112A05) and two opposing ionization probes for a total of sixteen sensors. These sensors are located at eight axial locations beginning at $1315 \mathrm{~mm}$ with a separation distance of $270 \mathrm{~mm}$. Both pressure and ionization probe signals are recorded with a high-speed acquisition system (NI, PXI-5105 Oscilloscope) at $30 \mathrm{MHz}$.

The combustible mixture used in this study is a quiescent hydrogenair mixture at near ambient conditions (1 bar and room temperature). The mixture is prepared using the partial pressures method utilizing a static pressure gauge (Keller Leo 3). The uncertainty in the equivalence ratio is within 3\%. Prior to the injection of the reactants, the tube is evacuated using a vacuum pump (Edwards RV8). The homogeneity of the hydrogenair mixture is obtained with the help of a recirculation pump (KNF Laboport N 842.3 FT.18).

The ignition is obtained by applying a high-voltage pulse of $6 \mathrm{kV}$, produced by a high-voltage power amplifier (Trek PD07016), to the spark plug. 
In order to change the ignition energy, the duration of the high-voltage pulse, $\tau$, as well as, the resistance of the spark plug may be varied. The ignition energy, $E_{\text {ign }}$, is determined by measuring the voltage $U$ and the current $I$ and using the following expression:

$$
E_{\mathrm{ign}}=\int_{0}^{\tau} U \times I d t
$$

All voltages are measured with high-voltage probes (Tektronix P6015A). The current is obtained by measuring the voltage drop across a $50 \Omega$ shunt resistor (Kanthal 887AS500KDG) connected in series with the spark plug on the ground side of the gap. The spark plug gap distance is set to $1 \mathrm{~mm}$. The three voltage signals are recorded using a high-speed oscilloscope (Agilent DSO9254A).

The NRP plasma discharges are produced by applying high-voltage pulses of $15 \mathrm{~ns}$ duration, at a repetition rate of $100 \mathrm{kHz}$. The maximum amplitude of the high-voltage pulses is $24.5 \mathrm{kV}$, produced by applying simultaneously a positive high-voltage pulse of $12.25 \mathrm{kV}$ and a negative high-voltage pulse of $-12.25 \mathrm{kV}$ to the electrodes with an NRP discharge generator (FID FPD 25-100MC2). The electrode configuration consists of a sharpened pin cathode (diameter $1.6 \mathrm{~mm}$, radius of curvature of the tip of about $100 \mu \mathrm{m}$ ) and a ring-shaped anode (inner diameter: $20 \mathrm{~mm}$, outer diameter: $24 \mathrm{~mm}$, thickness: $4 \mathrm{~mm}$ ) held in place by three stainless steel dowel pins, which are press fit through the wall of the acrylic glass tube. The pin electrode is positioned so that the sharpened tip is $5 \mathrm{~mm}$ upstream of the center point of the ring electrode, rather than being positioned in plane, resulting in a gap distance of around $11.2 \mathrm{~mm}$. Both electrodes are constructed out of stainless steel. The voltage at the electrodes is measured using high-voltage probes (Tek- 
tronix P6015A); and the current is measured using a Pearson current monitor (Model 6585). All voltage and current signals are recorded with a high-speed oscilloscope (Agilent DSO9254A) at a sampling rate of $2.5 \mathrm{GHz}$.

The NRP discharge energy, $E_{\mathrm{NRP}}$, is calculated analogously to the ignition energy, with the exception that the pulse duration is much shorter and the voltage and current signals must be carefully synchronized. This synchronization is realized by adjusting the temporal offset between the current and voltage signals such that $E_{\mathrm{NRP}}=0 \mathrm{~mJ}$ for an applied pulse that does not induce breakdown. The resulting uncertainty in the energy deposition for a single pulse is $\pm 100 \mu \mathrm{J}$. For plasma actuation, bursts from 35 to 150 NRP discharges have been used. The energy deposition by a burst of NRP discharges, $E_{\text {burst(NRP) }}$, is obtained by summing the energy of all individual pulses. The ignition, NRP discharge actuation, and measurement diagnostics are synchronized using a pulse/delay generator (Berkley Nucleonics Corporation, Model 575).

In order to ensure reproducible initial conditions, a careful experimental procedure is followed for these experiments. Before each experiment, the tube is purged with pressurized air for ten minutes in order to remove any excess water vapor from the previous experiment. Subsequently, the volume is evacuated to 10 mbar using the vacuum pump. Using partial pressures, the test bench is filled with a stoichiometric hydrogen-air mixture up to a pressure of 1 bar. The hydrogen and air are then mixed for three minutes using the recirculation pump, after which the mixture is given one minute to settle before being ignited. This experimental procedure and its repeatability were verified over the course of more than one hundred test runs before the NRP 
plasma experiments were conducted. The propagation speed of the reaction front is determined using the time-of-flight method from the ionization probe signals. An example of such signals as well as the pressure signals at the same axial location is provided in Fig. 2. This example shows a steady-state detonation wave with a propagation velocity near that of the Chapman-Jouguet (CJ) velocity of $1966 \mathrm{~m} / \mathrm{s}$. The primary source of uncertainty is due to the method of determining the time of arrival of the reaction wave. The arrival time is defined as the time at which the ionization probe signal drops to $99 \%$ of its initial value. This allows for the arrival event to be clearly identified above the inherent noise of the data acquisition system. Because the drop for a detonation wave is very steep, this uncertainty is $\pm 5 \mathrm{~m} / \mathrm{s}$. For the turbulent flame, the drop is more gradual leading to an uncertainty of $\pm 50 \mathrm{~m} / \mathrm{s}$.

Finally, in order to visualize the effect of NRP discharges on the flame propagation, the acrylic glass tube was replaced by a quartz glass tube of the same dimensions. The images are obtained with a high-speed CMOS camera (LaVision HSS8) coupled with an intensifier (LaVision IRO), a UV lens (105 mm F/5.6 Coastal Optics), and a 40-nm bandpass filter (LaVision 1108760) centered at $320 \mathrm{~nm}$, for selection of the $\mathrm{OH}^{*}$ chemiluminescence of the flame. The frame rate of the camera is synchronized with the plasma pulses at one frame per pulse. The exposure time is $9 \mu \mathrm{s}$. Note that the light emission from the discharges is collected simultaneously with the flame emission. Therefore, in the location of the discharges the light emission is not only due to the $\mathrm{OH}(\mathrm{A}-\mathrm{X})$ transition but also to the $\mathrm{N}_{2}(\mathrm{C}-\mathrm{B})$ transition, excited nitrogen being one of the main emitting species of non-equilibrium discharges in this range of wavelengths. 


\section{Results}

\subsection{Combustion front propagation without plasma actuation}

A common way to ignite combustible mixtures is to use spark discharges. Typically, these sparks are produced by applying high-voltage pulses of several kilovolts, with durations of $10 \mu \mathrm{s}$ to $5 \mathrm{~ms}$, between two electrodes separated by a gap distance from $500 \mu \mathrm{m}$ to a few millimeters. The type of plasma produced can usually be characterized as an arc discharge [32]. By varying the duration of the applied voltage and the features of the electric field, for a same energy deposition, the ignition of the combustible mixture can be completely different (e.g., [33-35, 31]). In the present study, the effect of the characteristics of the ignition source on DDT is not investigated. The ignition energy has only been slightly varied in order to be in the same range as the electrical energy deposited by the NRP discharges. An ignition system that mimics that of an automobile engine has been chosen.

Table 1: Summary of the five ignition conditions tested. The duration, $\tau$, of the ignition pulse and the built-in resistance of the spark plug control the ignition energy, $\mathrm{E}_{\text {ign }}$.

\begin{tabular}{cccccc}
\hline Ignition condition & 1 & 2 & 3 & 4 & 5 \\
Spark plug resistance, $\mathrm{k} \Omega$ & 4.6 & 0 & 4.6 & 0 & 4.6 \\
$\tau, \mathrm{ms}$ & 1 & 1.5 & 2 & 2 & 4 \\
$E_{\mathrm{ign}}, \mathrm{mJ}$ & 9 & 52 & 20 & 70 & 45 \\
\hline
\end{tabular}

The baseline ignition spark is obtained using a spark plug with a builtin resistance of $4.6 \mathrm{k} \Omega$, to which a $6 \mathrm{kV}$ pulse with a duration of $\tau=1 \mathrm{~ms}$ is applied. Although $6 \mathrm{kV}$ is applied, breakdown occurs consistently at just 
over $4 \mathrm{kV}$ for these conditions. After breakdown, the ignition system supplies sufficient power for just over $20 \mu$ s at a constant current of $350 \mathrm{~mA}$, according to the specifications of the device. After this plateau, the supply current collapses and the system exhibits an oscillating behavior periodically depositing energy throughout the remainder of the pulse duration. In this way, energy deposition for the ignition spark amounts to $9 \pm 2 \mathrm{~mJ}$. An example of the associated voltage and current signals as well as the energy deposition with respect to time for this $1 \mathrm{~ms}$ pulse is given in Fig. 3. Increasing the duration of the pulse effectively increases the energy deposition of ignition spark, $\mathrm{E}_{\mathrm{ign}}$. Furthermore, installing the non-resistive spark plug allows for even higher energy deposition. Table 1 summarizes the various ignition conditions tested in the course of this study.

As expected for such low energies, the occurrence of DDT is not observed for any of these ignition conditions. Furthermore, no apparent effect is evident on the initial flame propagation. In fact, the variation due to the stochastic character of turbulent flame propagation significantly overshadows any possible effect that the ignition energy in the specified range of conditions may have on the flame propagation. This is depicted for example test cases in Fig. 4. Notice that there are two modes of propagation regardless of ignition energy. One is characterized by extreme deceleration of the flame towards the end of the tube (dashed lines in Fig. 4). This is likely due to the "tulip flame" phenomenon, which is known to readily occur in propagating flames in tubes [36]. The other is characterized by the flame successfully resisting these oscillations and continuing to moderately accelerate to speeds of around $1000 \mathrm{~m} / \mathrm{s}$ (solid lines in Fig. 4). On average, regardless of the 
ignition energy (in the range investigated), in about $60 \%$ of the cases, the flame decelerates, while in $40 \%$ of the measurements, the flame accelerates. It is important to note that these tests are conducted with the electrodes shown in Fig. 1 installed. Therefore, although there is no plasma actuation, the fluid dynamic effects on turbulence generation, which also accelerate the flame, are identical to the tests with plasma actuation.

\subsection{Combustion front propagation with NRP discharges}

Electrical measurements of the NRP plasma discharges during flame actuation indicate that the discharges are in the NRP spark regime [23]. However, for an applied voltage of $24.5 \mathrm{kV}$, occurrence of weak and strong NRP sparks is observed. An example of voltage, total current, and energy deposition for a weak NRP spark discharge is shown in Fig. 5a. The weak NRP spark discharges exhibit a maximum current of around $27 \mathrm{~A}$ and energy deposition of around $1 \pm 0.1 \mathrm{~mJ}$ per pulse. Assuming a discharge diameter of $0.8 \mathrm{~mm}$ and a discharge length of $11.2 \mathrm{~mm}$, the energy density is then about $0.178 \mathrm{~J} /\left(\mathrm{cm}^{3} \cdot \mathrm{bar}\right)$. In contrast, the strong NRP sparks exhibit maximum currents in excess of $100 \mathrm{~A}$. The energy per pulse is then around $11 \pm 0.2 \mathrm{~mJ}$ $\left(1.96 \mathrm{~J} /\left(\mathrm{cm}^{3} \cdot\right.\right.$ bar $\left.)\right)$, approaching the design maximum energy per pulse for the power supply of $12 \mathrm{~mJ}$. An example of voltage, total current, and energy deposition for a strong NRP spark discharge is shown in Fig. 5b.

When a burst of pulses is applied, the initial pulses always begin in the weak spark regime and at some point, they may transition to the strong sparks. This may occur as early as pulse 20-30 or may not occur at all. If the strong NRP spark regime occurs too early, it may ignite the mixture independently and disrupt the acceleration of the flame. In addition, for a 
given number of pulses, it will drastically increase the energy deposition. For these reasons, the occurrence of strong NRP sparks must be controlled and, if possible, avoided. In order to do so, the total voltage between the electrodes is limited to $24.5 \mathrm{kV}$. In addition, as the probability of the transition to the strong NRP sparks increases with the number of pulses, decreasing the number of pulses should also be considered. Table 2 summarizes the different conditions of NRP discharge actuation that have been evaluated in this study.

Table 2: Summary of the conditions of NRP discharge actuation tested. For all of these conditions, the applied voltage is $24.5 \mathrm{kV}$, the pulse repetition frequency is $100 \mathrm{kHz}$, and the energy of the ignition spark is $9 \mathrm{~mJ}$.

\begin{tabular}{cccc}
\hline Number of pulses & 150 & 100 & 35 \\
\hline Duration of plasma actuation, ms & 1.5 & 1 & 0.35 \\
\hline Minimum observed $E_{\text {burst (NRP) }}$, mJ & 150 & 100 & 35 \\
\hline$E_{\text {burst (NRP) }}+E_{\text {ign }}, \mathrm{mJ}$ & 159 & 109 & 44 \\
\hline
\end{tabular}

Careful timing between ignition and plasma actuation is necessary. In order to observe the maximum effect on the combustion front propagation speed, NRP discharges must be applied when the flame is in the vicinity of the electrodes. Applying the burst of NRP discharges too long before or after the flame passes through the electrode assembly results in no effect on the flame acceleration. As it can be observed in Fig. 4, the speed of the flame can vary from 200 to $700 \mathrm{~m} / \mathrm{s}$ when it enters the measurement section. Based on the time of arrival of the flame at the first ionization probe and its propagation 
speed at this location, it is estimated that the flame consistently arrives at the electrodes at between $11.5 \mathrm{~ms}$ and $12.5 \mathrm{~ms}$ after ignition. For a pulse train of 150 pulse at a repetition frequency of $100 \mathrm{kHz}$ and beginning the NRP pulses after a delay of $11 \mathrm{~ms}$ after ignition ensures repeatable DDT. Systematically increasing the delay and decreasing the number of pulses allows for DDT to be achieved with as little as 35 pulses. Due to the jitter in the arrival of the flame front, however, reducing the number of pulses has a direct impact on the repeatability of the experiments.

The effect of plasma actuation obtained for a burst of 100 pulses and for a delay between the ignition and the plasma actuation of $11 \mathrm{~ms}$ is presented in Fig. 6, along with cases for no plasma actuation. Plasma actuation consistently results in the occurrence of DDT. In order to confirm the reliability of using these NRP plasma discharges in accelerating DDT, a series of twenty test runs alternating between plasma actuation and no plasma actuation were conducted using an ignition energy of $9 \mathrm{~mJ}$. In the case of no plasma actuation, DDT was never observed. In eight of ten cases, the flame exhibited the aforementioned extreme deceleration. This number is slightly higher than the $60 \%$ of decelerating flames observed for the investigation of the effect of ignition energy (see Section 3.1). This is probably due to the limited number of tests. In the cases with NRP discharge actuation, a significant effect is observed on the flame acceleration and DDT occurred in all cases. The flame velocity observed between the second and third ionization probes is already more than double that of the tests without plasma.

Figure 7 compares the reaction front propagation speeds obtained for plasma actuation with 150 (blue), 100 (red) and 35 (black) pulses. For the 
three cases, DDT occurs at about $3 \mathrm{~m}$. The effect of the NRP discharges seems to be not strongly correlated to the number of pulses. However, as it can be noticed in Fig. 6, where 10 tests performed with a burst of 100 pulses are depicted, that the variation in the acceleration of the flame front can be large. For example, in five cases the transition to detonation has already occurred at $2.5 \mathrm{~m}$, while for the other five cases, the propagation speed is less than $1500 \mathrm{~m} / \mathrm{s}$. Further investigation will be needed to make any conclusion about the effect of the number of pulses on the flame acceleration.

In order to better understand the mechanism of plasma actuation on the flame acceleration, high-speed visualization of the $\mathrm{OH}^{*}$ chemiluminescence has been done. An example of images obtained for a burst of 35 pulses is presented in Fig. 8. The delay between images is roughly $40 \mu$ s. In Fig. 8a, corresponding to a delay of about $11.98 \mathrm{~ms}$ after ignition, the light emission from the discharge produced by the $10^{\text {th }}$ pulse of the burst is barely visible and only at the tip of the cathode. This corresponds to an energy deposition of about $1.1 \mathrm{~mJ}$ and is in the weak NRP spark regime. On the left, the flame enters the visualization area. Four pulses later (Fig. 8b), the flame has traveled closer to the inter-electrode area, but still the discharge occurs in the fresh gases. Obviously, pulses 1 to 14 have been produced in the fresh gases and they did not ignite the hydrogen-air mixture. As the flame reaches the tip of the cathode (Fig. 8c), a bright filament can be observed, superimposed with the flame. The light from the discharge is visible along the entire gap. In Fig. $8 \mathrm{~d}$ and $8 \mathrm{e}$, corresponding to the $22^{\text {nd }}$ and $26^{\text {th }}$ pulses, the light from the discharges generated in the burnt gases is strong enough to saturate the camera. This corresponds to the strong NRP discharge regime. Based on 
flame tracking, the propagation velocity of the flame before it reaches the electrodes is $200 \pm 20 \mathrm{~m} / \mathrm{s}$. After interaction with the plasma, this velocity is around $410 \pm 20 \mathrm{~m} / \mathrm{s}$. This supports the observation of the doubling of the propagation speed at the first ionizations probes when NRP discharges are applied.

During the course of these experiments, it was determined that it is possible of obtain DDT with only a train of 35 weak discharges (as shown in Fig. 5a). However, it is important to emphasize the fact that successful synchronization of such a train is very difficult. The typical course of events is that the pulses begin as weak discharges in the fresh gases and as the flame enters the inter-electrode area, the discharge transitions to the strong regime due to the change in the reduced electric field. For example, the images shown in Fig. 8 correspond to one such case. Only if the pulses are stopped before the flame enters the inter-electrode area, can DDT be obtained without strong discharges. The maximum allowable time delay between ceasing of the discharge pulses and flame arrival is not yet known, but from our preliminary results, it should be on the order of a few hundred microseconds. Due to the stochastic nature of the flame, this parameter has not been investigated in the current setup.

These tests establish proof of concept for using NRP plasma discharges in enhancing flame acceleration and decreasing DDT run-up length. Furthermore, this may be accomplished only with discharges in the weak spark regime if the last pulse occurs shortly before the flame arrives. For this case, the total required energy for successful DDT, the sum of the ignition spark and the $35 \mathrm{NRP}$ spark discharges, is around $44 \mathrm{~mJ}$ (see Fig. 3 and Fig. 5). 
However, it is important to note that synchronization is difficult without a triggering scheme due to the stochastic nature of the flame propagation. Consequently, using only 35 pulses may result in no DDT if the necessary conditions are not met. Nevertheless, keeping in mind that transition to detonation could not be observed for an ignition energy up to $70 \mathrm{~mJ}$, this strategy of flame acceleration by NRP discharges could be energetically more efficient than increasing the energy of the ignition source.

\section{Discussion}

The effect of plasma actuation on DDT is assessed by applying a burst of NRP discharges synchronized with the propagation of a stoichiometric hydrogen-air flame in the inter-electrode area. The stochastic nature of the flame propagation leads invariably to the first pulses being applied in the fresh mixture. The following pulses may be applied to the flame itself or even in the burnt gases. Due to the strong shot-to-shot jitter in the flame propagation speed, it is difficult to predetermine how many of these pulses will be applied to each of these domains. It is possible to determine this a posteriori by using a high-speed visualization system such as an intensified camera with a frame rate of at least $100 \mathrm{kHz}$. Based on the example presented in Fig. 8, where it is shown that about 15 discharges are generated in the

fresh gases, about 5 discharges in the flame front, and the remaining 15 in the burnt gases, it is worthwhile to discuss what their relative effect on the flame acceleration could be.

The pulses applied ahead of the flame front do not ignite the hydrogen-air mixture. This is visible in Figs. 8a and 8b, and in addition, it has been verified 
that applying a burst of 35 pulses to the fresh mixture, for which no ignition source was used $\left(E_{\text {ign }}=0 \mathrm{~mJ}\right)$, did not ignite the mixture. The pulses either do not lead to the breakdown of the mixture or produce weak NRP sparks with an energy of $E_{\mathrm{NRP}}=1 \mathrm{~mJ}$, which are too weak to ignite the mixture. The measured $E_{\mathrm{NRP}}$ is significantly higher than the minimum ignition energy (MIE) for such a mixture, which is around $0.1 \mathrm{~mJ}$ for a gap distance of $3 \mathrm{~mm}$, as measured by Ono et al. [37]. However, when extrapolating their results to a gap distance of $11.2 \mathrm{~mm}$, the MIE would then be $0.373 \mathrm{~mJ}$. Furthermore, it is very common for mixtures not to ignite for energies up to three times the MIE (i.e., $1.1 \mathrm{~mJ}$ [37]), for non-optimized geometry and material of the electrodes. If the weak NRP discharges applied to the fresh combustible mixture are not able to ignite a flame, they can nevertheless have a strong local thermal and chemical impact on the fresh gases, as shown in pure air, for example in $[25,38]$. The NRP spark discharges are known to heat the gas in an ultra-fast manner (a few tens of nanoseconds), up to a few thousands of kelvin, and to dissociate up to $50 \%$ of the oxygen [24]. In the present study, the thermal and chemical impact of the weak NRP sparks is probably less than the maximum values measured in air. Nevertheless, the weak NRP sparks produced in the fresh gases should locally increase the combustion rate, increasing the wrinkling of the turbulent flame, and globally accelerating the combustion front.

The pulses applied when a part of the flame front is in the inter-electrode gap (see, for example, Fig. 8c), are likely to be the most efficient in enhancing the flame acceleration. First, the presence of a reaction front at high-temperature increases the local reduced electric field, which promotes 
the ionization and electronic excitation of the molecules [39, 40]. Consequently, compared to discharges in the fresh gases, the plasma produced by the NRP sparks when a flame front is in the inter-electrode gap will have a stronger thermal and chemical impact. Also, when the NRP discharges are applied directly across the flame, the plasma produced does not relax into a less reactive medium before interacting with the combustion front.

The pulses applied in the burnt gases may also have an impact on the acceleration of the flame. As it can been seen in Figs. 8d and 8e, the discharges produced can be intense. The NRP discharges should then result in a significant ultra-fast gas heating that will induce the propagation of shock and pressure waves [41], which propagate into both the fresh gases and the burnt gases. Therefore, the phenomenon of shock-flame interaction could have a significant impact on the flame acceleration. When the shock waves produced by the discharges interact with the flame, it can result in a wrinkling of the flame due to the Richtmyer-Meshkov instability. This phenomenon is due to the impulsive acceleration of the relatively discontinuous interface between the unburnt and burnt gases. In contrast to the Rayleigh-Taylor instability, which is responsible for the "tulip flame," the Richtmyer-Meshkov instability is unstable in both directions [42]. In other words, it is irrelevant whether the shock waves from the discharge travel upstream through the fresh gases to meet the flame or downstream through the burnt gases to meet the flame. This means that every individual discharge that is strong enough to generate a shock wave has the potential of increasing flame wrinkling. The cumulative effect of several discharges may significantly enhance the combustion through flame wrinkling alone even for relatively weak indi- 
vidual shock waves. This effect has been observed by Thomas et al. [43] for a single shock wave. For strong incident shock waves, they observed DDT shortly after the shock interacted with a spherically expanding flame. Although the shock wave was much stronger and the flame much slower than in the present work, the shock-flame interaction may also contribute to the observed flame acceleration induced by NRP discharge actuation.

Finally, it is interesting to compare the electrical power of the plasma actuation with the thermal power of the flame. The average electrical power applied during NRP discharge actuation can be obtained by multiplying the energy deposition during a single pulse by the number of pulses during a second. As the pulse repetition frequency is fixed at $100 \mathrm{kHz}$, the average plasma power is in the range from $100 \mathrm{~W}$, for the weak NRP sparks, to $1.1 \mathrm{~kW}$, for the strong NRP sparks. Considering the initial conditions (stoichiometric hydrogen-air mixture at 1 bar and room temperature), the lower heating value of hydrogen $(120 \mathrm{MJ} / \mathrm{kg})$, and the diameter of the tube, the heat release of the flame per unit length is $3.44 \mathrm{~kJ} / \mathrm{m}$. When the flame enters the interelectrode area, its velocity is typically in the range of 200 to $700 \mathrm{~m} / \mathrm{s}$ (see Fig. 4). Consequently, the thermal power of the flame in the plasma area is in the range of $862 \mathrm{~kW}$ to $2.4 \mathrm{MW}$. Thus, the percentage of NRP discharge electrical power with respect to the thermal power of the flame is in the range of 0.004 to $0.14 \%$.

These values are more than one order of magnitude lower than the usual ratio of plasma power to flame thermal power that is used for enhancing flame properties, such as decreasing the lean blow-off limit [44], or controlling thermoacoustic instabilities [45]. The main difference is that in all of 
these previous studies, the flames were stabilized and not freely propagating. In fact, freely propagating flames are usually more sensitive to small perturbations than stabilized flames. The necessary run-up length to DDT for practical PDC applications, however, is significantly lower than what has been obtained in this study. In order to achieve such lengths, it is likely that the electrical power of the NRP discharges must be higher. Nevertheless, a plasma actuator using NRP discharges might be a realistic alternative to passive flame accelerators.

\section{Conclusions}

Proof of concept has been achieved for the use of NRP discharges during the flame acceleration phase in order to achieve a significantly decreased run-up length to DDT. These findings have been obtained using time-offlight measurements. The energy deposition of both the ignition spark and the NRP discharges have been quantified. Increasing only the ignition energy from $9 \mathrm{~mJ}$ to $70 \mathrm{~mJ}$ results in no observable effect on the flame acceleration. However, using a low-energy spark ignition $(9 \mathrm{~mJ})$ in combination with a properly-timed train of $35 \mathrm{NRP}$ discharges results in significant flame acceleration and subsequent DDT for an electrical energy as low as $44 \mathrm{~mJ}$. This decrease in the run-up length to DDT is obtained for a plasma power of less than $0.14 \%$ of the thermal power of the flame. High-speed visualization of the flame $\mathrm{OH}^{*}$ chemiluminescence shows that just after plasma actuation, the flame propagates two times faster than before plasma actuation. Further study will be necessary in order to determine the main mechanisms of the effect of the NRP discharges on the flame acceleration. Nevertheless, this 
strategy presents not only a more energetically efficient alternative to simply increasing the energy deposition in the ignition spark, but also a promising strategy for reducing or replacing obstacles in pulse detonation applications.

\section{Acknowledgements}

This work is funded by the Center of Competitive Funding from King Abdullah University of Science and Technology (Grant Number 1975).

\section{References}

[1] R. Zitoun, D. Desbordes, Propulsive performances of pulsed detonations, Combustion Science and Technology 144(1-6) (1999) 93-114.

[2] D. Paxson, T. Kaemming, Influence of unsteadiness on the analysis of pressure gain combustion devices, Journal of Propulsion and Power 30 (2) (2014) 377-383.

[3] S. Frolov, V. Aksenov, V. Ivanov, Experimental proof of Zel'dovich cycle efficiency gain over cycle with constant pressure combustion for hydrogen-oxygen fuel mixture, International Journal of Hydrogen Energy 40 (21) (2015) 6970-6975.

[4] J. Gray, J. Vinkeloe, C. Paschereit, P. Stathopoulos, P. Berndt, R. Klein, Thermodynamic evaluation of pulse detonation combustion for gas turbine power cycles, in: ASME Turbo Expo, 2016.

[5] R. Blackburn, R. Miller, The Rayleigh efficiency of pressure gain combustors, Journal of Propulsion and Power 33 (1) (2017) 51-61. 
[6] J. Lisanti, W. Roberts, Pulse Combustor Driven Pressure Gain Combustion for High Efficiency Gas Turbine Engines. In. Agarwal A., De S., Pandey A., Singh A. (eds). Combustion for Power Generation and Transportation., Springer, Singapore, 2017.

[7] T. Jacobsson, V. Fjallstrom, M. Edoff, T. Edvinsson, Cigs based devices for solar hydrogen production spanning from pec-cells to pvelectrolyzers: A comparison of efficiency, stability and device topology, Solar Energy Materials and Solar Cells 134 (2015) 185-193.

[8] D. Zhao, C. Yang, Recent advances in the tio2/cds nanocomposite used for photocatalytic hydrogen production and quantum-dot-sensitized solar cells, Renewable and Sustainable Energy Reviews 54 (2016) 10481059.

[9] D. Ferrero, M. Santarelli, Investigation of a novel concept for hydrogen production by pem water electrolysis integrated with multi-junction solar cells, Energy Conversion and Management 148 (2017) 16-29.

[10] W. Fickett, W. Davis, Detonation Theory and Experiment, Chapter 2, Dover, Mineola, New York, 2001.

[11] J. Lee, The Detonation Phenomenon, Chapter 8, Cambridge University Press, U.K., 2008.

[12] J. Lee, R. Knystautas, C. Chan, Turbulent flame propagation in obstacle-filled tubes, Symposium (International) on Combustion 20 (1985) 1663-1672. 
[13] F. Schauer, J. Stutrud, R. Bradley, Detonation initiation studies and performance results for pulsed detonation engine applications, in: 39th AIAA Aerospace Sciences Meeting and Exhibit, 2001.

[14] G. Ciccarreli, S. Dorofeev, Flame accleration and transition to detonation in ducts, Progress in Energy and Combustion Science 34(4) (2008) 499-550.

[15] R. Scarpa, E. Struder, B. Cariteau, S. Kudriakov, N. Chaumeix, Ir absorption measurements of the velocity of a premixed hydrogen/air flame propagating in a obstacle-laden tube, in: 26th International Colloquium on the Dynamics of Explosions and Reactive Systems, 2017.

[16] D. Paxson, T. Kaemming, Performance Impact of Deflagration to Detonation Transition Enhancing Obstacles, in: 37th AIAA Aerospace Sciences Meeting and Exhibit, 2009.

[17] S. Frolov, V. Basevich, V. Aksenov, S. Polikhov, Detonation initiation by controlled triggering of electric discharges, Journal of Propulsion and Power 19 (4) (2003) 573-580.

[18] S. Frolov, V. Basevich, V. Aksenov, S. Polikhov, Optimization study of spray detonation initiation by electric discharges, Shock Waves 14 (3) (2005) 175-186.

[19] V. Kamenskihs, J. Lee, Effect of an axial electric field on detonation waves, Combustion and Flame 159 (2012) 2967-2973.

[20] S. Starikovskaia, Plasma assisted ignition and combustion, Journal of Physics D: Applied Physics 39 (2006) R265-R299. 
[21] Y. Ju, W. Sun, Plasma assisted combustion: Dynamics and chemistry, Progress in Energy and Combustion Science 48 (2015) 21-83.

[22] D. Pai, G. Stancu, D. Lacoste, C. Laux, Nanosecond repetitively pulsed discharges in air at atmospheric pressure - the glow regime, Plasma Sources Science and Technology 18 (2009) 045030(7pp).

[23] D. Pai, D. Lacoste, C. Laux, Nanosecond repetitively pulsed discharges in air at atmospheric pressure - the spark regime, Plasma Sources Science and Technology 19 (2010) 065015(10pp).

[24] G. Stancu, F. Kaddouri, D. Lacoste, C. Laux, Atmospheric pressure plasma diagnostics by oes, crds and talif, Journal of Physics D: Applied Physics 43 (2010) 124002(10pp).

[25] D. Rusterholtz, D. Lacoste, G. Stancu, D. Pai, C. Laux, Ultrafast heating and oxygen dissociation in atmospheric pressure air by nanosecond repetitively pulsed discharges, Journal of Physics D: Applied Physics 46 (2013) 464010(21pp).

[26] V. Zhukov, A. Starikovskii, Effect of a nanosecond gas discharge on deflagration to detonation transition, Combustion Explosion and Shock Waves 42 (2) (2006) 195-204.

[27] K. Busby, J. Corrigan, S. Yu, S. Williams, C. Carter, F. Schauer, J. Hoke, C. Cathey, M. Gundersen, Effects of corona, spark and surface discharges on ignition delay and deflagration-to-detonation times in pulsed detonation engines, in: 45th AIAA Aerospace Sciences Meeting and Exhibit, 2007. 
[28] A. Rakitin, A. Starikovskii, Mechanisms of deflagration-to-detonation transition under initiation by high-voltage nanosecond discharges, Combustion and Flame 155 (1-2) (2008) 343-355.

[29] V. Zhukov, A. Rakitin, A. Starikovskii, Effect of high-voltage pulsed discharges on deflagration to detonation transition, Journal of Propulsion and Power 24 (1) (2008) 88-93.

[30] A. Starikovskiy, N. Aleksandrov, A. Rakitin, Plasma-assisted ignition and deflagration-to-detonation transition, Phil. Trans. R. Soc. A 370 (2012) 740-773.

[31] J. Lefkowitz, P. Guo, T. Ombrello, S. Won, C. Stevens, J. Hoke, F. Schauer, Y. Ju, Schlieren imaging and pulsed detonation engine testing of ignition by a nanosecond repetitively pulsed discharge, Combustion and Flame 162 (6) (2015) 2496-2507.

[32] Y. Raizer, Gas Discharge Physics, Chapter 10, Springer, Berlin, Heidelberg, 1991.

[33] D. Ballal, A. Lefebvre, Influence of spark discharge characteristics on minimum ignition energy in flowing gases, Combustion and Flame 24 (1) (1975) 99-108.

[34] S. Shy, C. Liu, W. Shih, Ignition transition in turbulent premixed combustion, Combustion and Flame 157 (2010) 341-350.

[35] Q. Zhang, W. Li, S. Zhang, Effects of spark duration on minimum ignition energy for methane/air mixture, Process Safety Progress 30 (2) (2011) 154-156. 
[36] C. Clanet, Searby, G., On the Tulip Flame Phenomenon, Combustion and Flame 105 (1996) 225-238.

[37] R. Ono, M. Nifuku, S. Fujiwara, S. Horoguchi, T. Oda, Minimum ignition energy of hydrogenair mixture: Effects of humidity and spark duration, Journal of Electrostatics 65 (2007) 87-93.

[38] A. Lo, A. Cessou, P. Boubert, P. Vervisch, Space and time analysis of the nanosecond scale discharges in atmospheric pressure air: I. gas temperature and vibrational distribution function of n-2 and o-2, Journal of Physics D: Applied Physics 47 (11) (2014) 115201.

[39] W. Nighan, Electron energy distributions and collision rates in electrically excited n2, co, and co2, Physical Review A 2 (5) (1970) 1989-2000.

[40] N. Aleksandrov, F. Vysikailo, R. Islamov, I. Kochetov, A. Napartovich, V. Pevgov, Electron-distribution function in 4-1 n-2-o-2 mixture, High Temperature 19 (1) (1981) 17-21.

[41] D. Xu, D. Lacoste, D. Rusterholtz, P. Elias, G. Stancu, C. Laux, Experimental study of the hydrodynamic expansion following a nanosecond repetitively pulsed discharge in air, Applied Physics Letters 99 (2011) 121502.

[42] R. Krechetnikov, Rayleigh-taylor and richtmyer-meshkov instabilities of flat and curved interfaces, Journal of Fluid Mechanics 625 (2009) 387-410.

[43] G. Thomas, R. Bambrey, C. Brown, Experimental observations of flame 
acceleration and transition to detonation following shock-flame interaction, Combustion Theory and Modelling 5 (2001) 573-594.

[44] G. Pilla, D. Galley, D. Lacoste, F. Lacas, D. Veynante, C. Laux, Stabilization of a turbulent premixed flame using a nanosecond repetitively pulsed plasma, IEEE Transactions on Plasma Science 34 (6) (2006) 2471-2477.

[45] J. Moeck, D. Lacoste, C. Laux, C. Paschereit, Control of combustion dynamics in a swirl-stabilized combustor with nanosecond repetitively pulsed discharges, in: 51st AIAA Aerospace Sciences Meeting including The New Horizons Forum and Aerospace Exposition, 2013. 


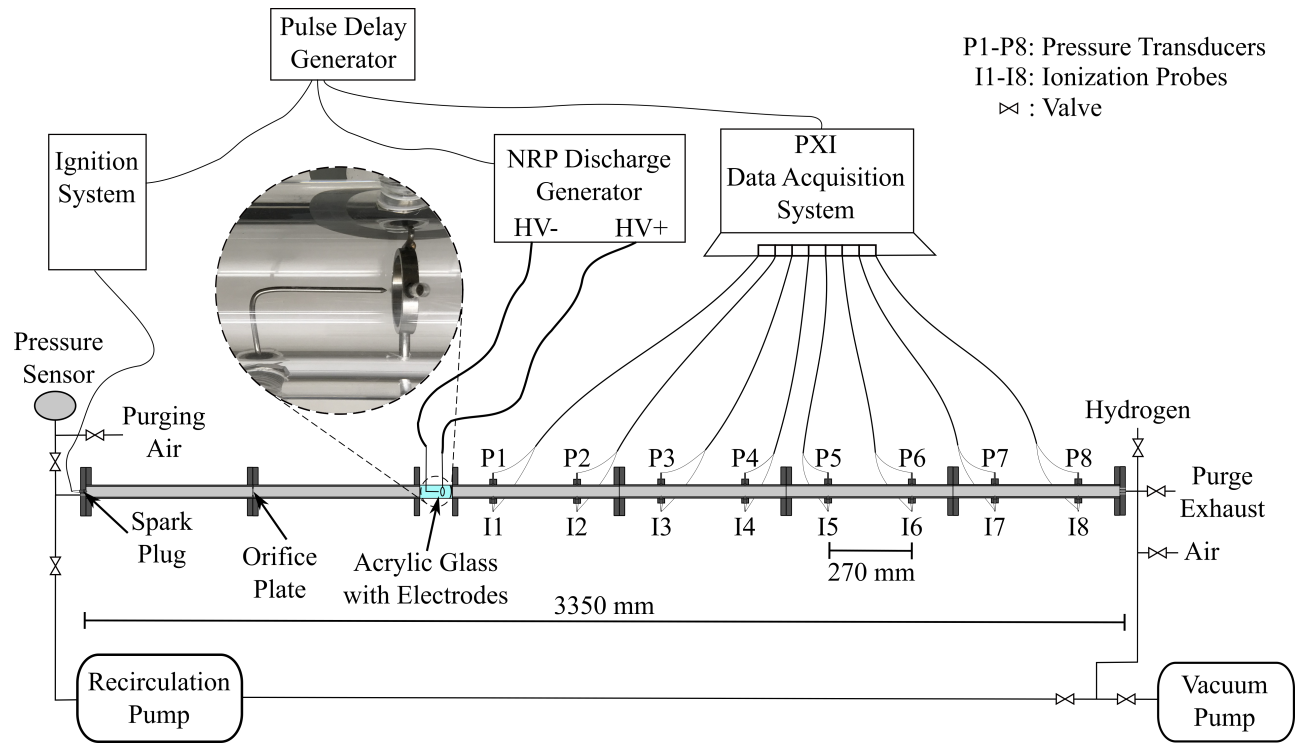

Figure 1: Schematic representation of the experimental test bench including a photograph detailing the electrode configuration. 


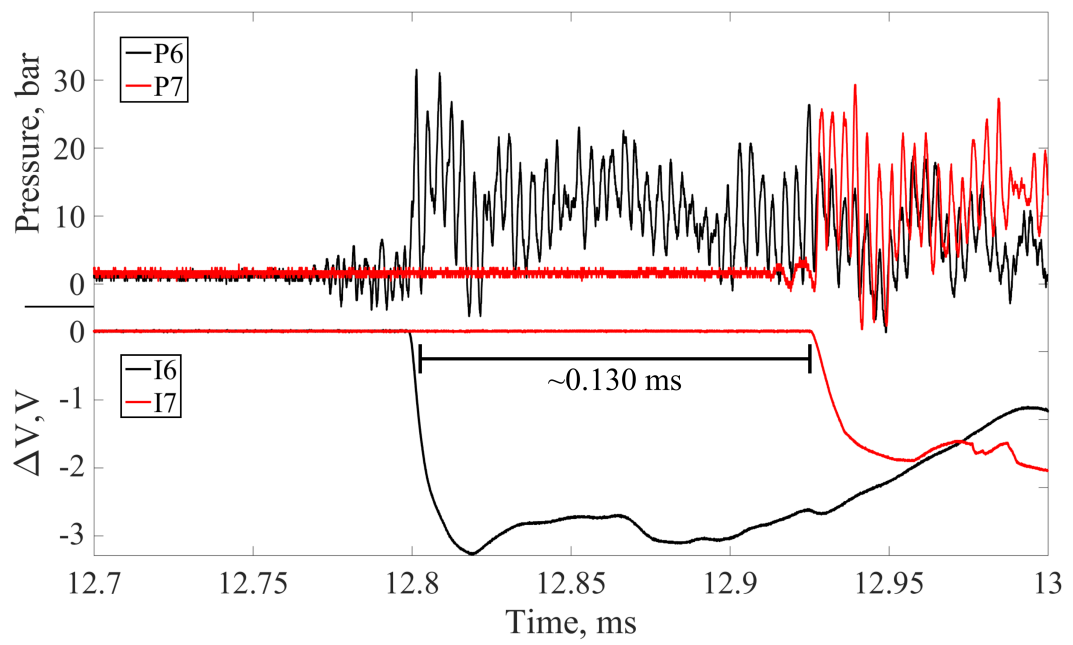

Figure 2: Example waveforms obtained from piezoelectric pressure transducers and ionization probes at two axial positions. The positions for P6, P7, I6, and I7 are shown in Fig. 1. These signals are used to obtain the time-of-flight for the reaction waves. A time of around $0.130 \mathrm{~ms}$ corresponds roughly to CJ velocity. 


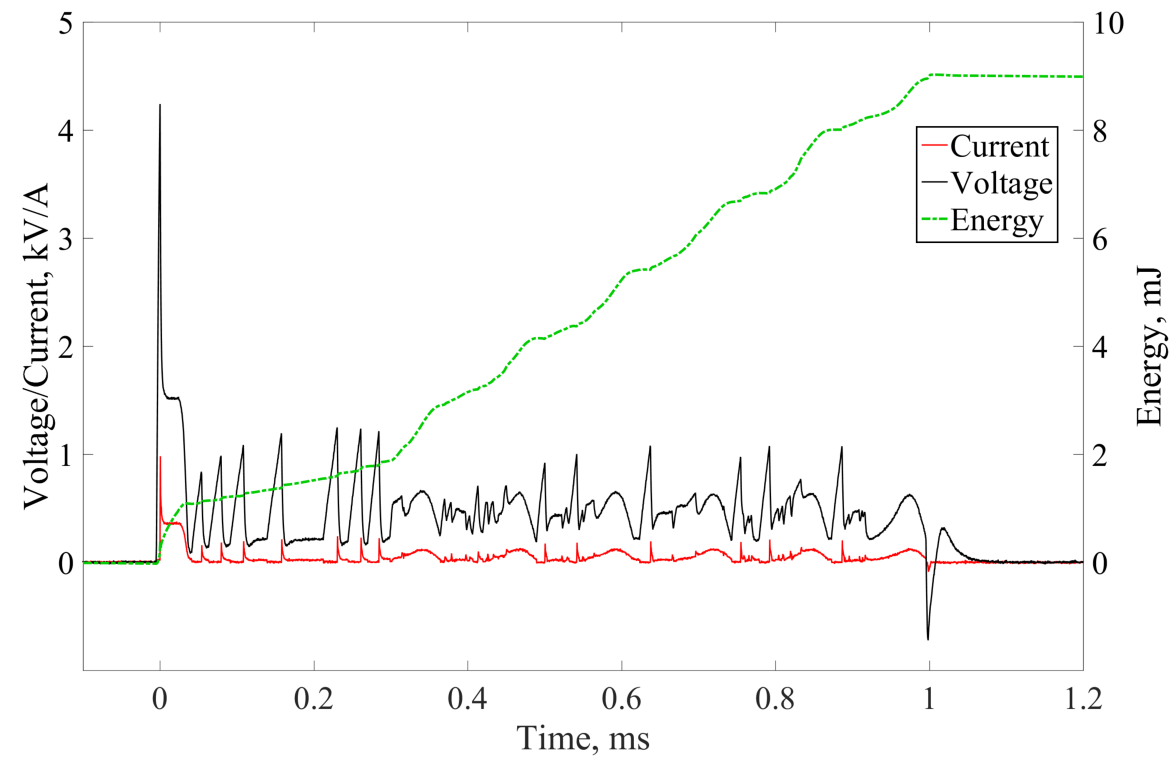

Figure 3: Example of waveforms for voltage and total current as well as the corresponding calculated energy deposition for an ignition spark with a duration of $\tau=1 \mathrm{~ms}$. The oscillations following the initial breakdown and plateau are due to a power limitation of the power supply. 


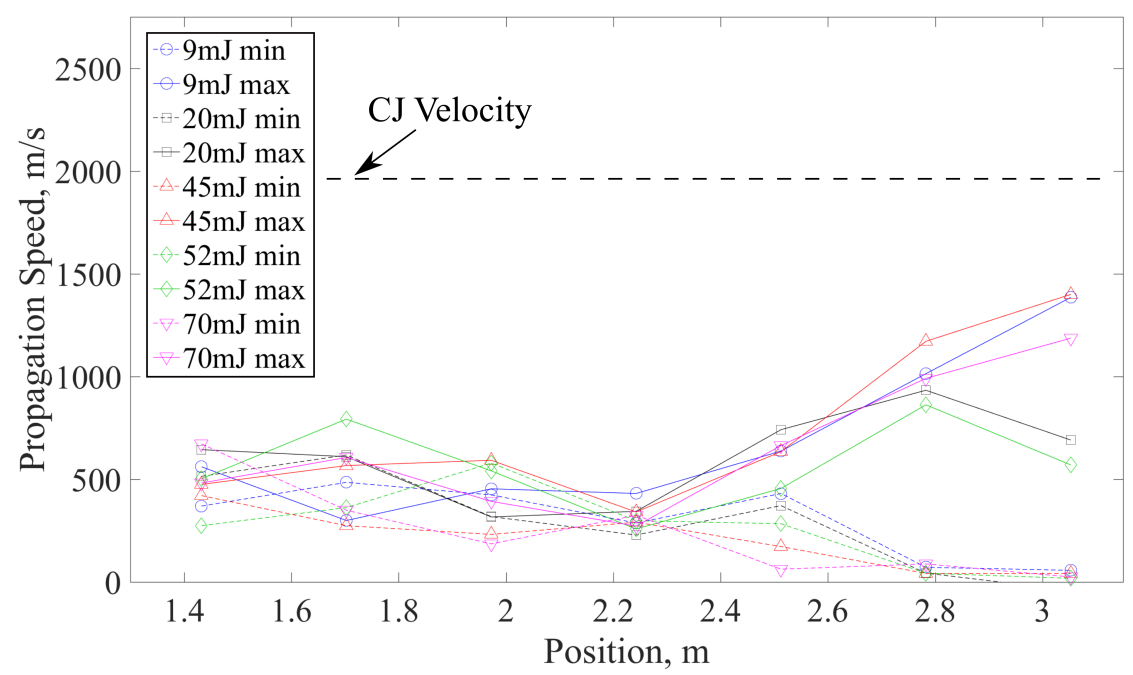

Figure 4: Development of the reaction front propagation speed obtained from time-offlight measurements using ionization probes for cases with varying ignition energies with no NRP plasma discharges. The uncertainty in these velocity measurements is $\pm 50 \mathrm{~m} / \mathrm{s}$. 


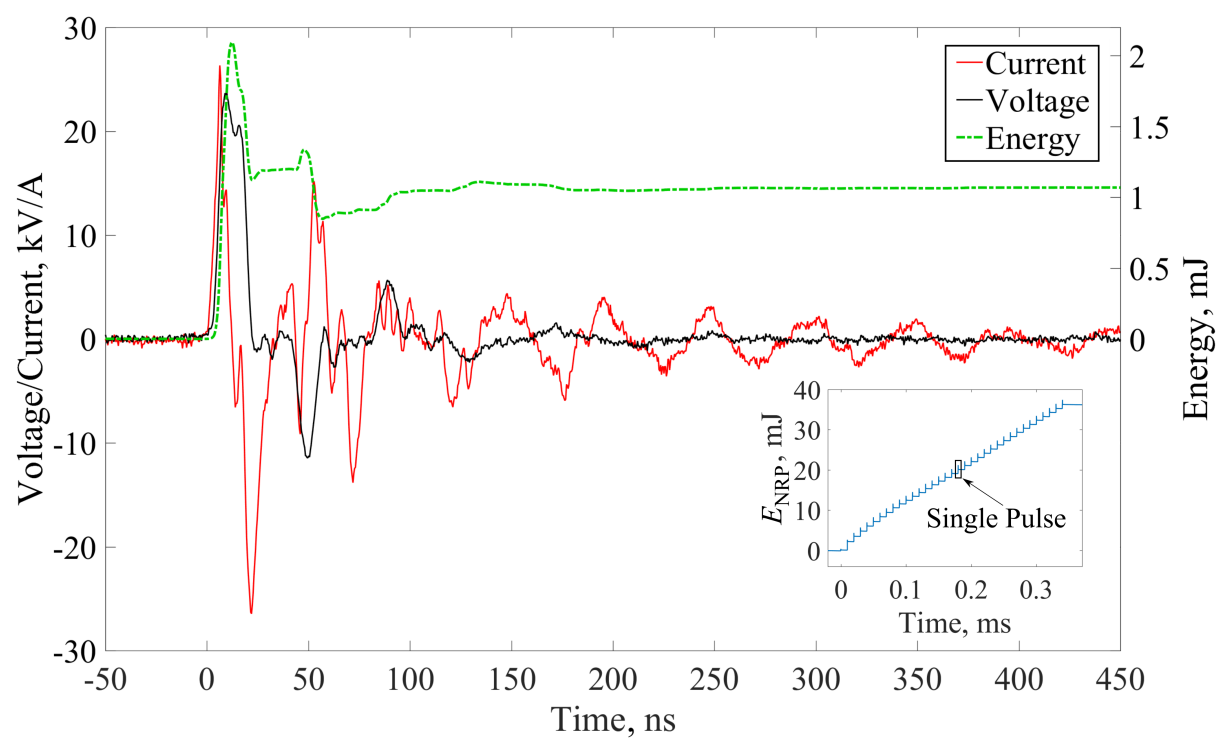

(a)

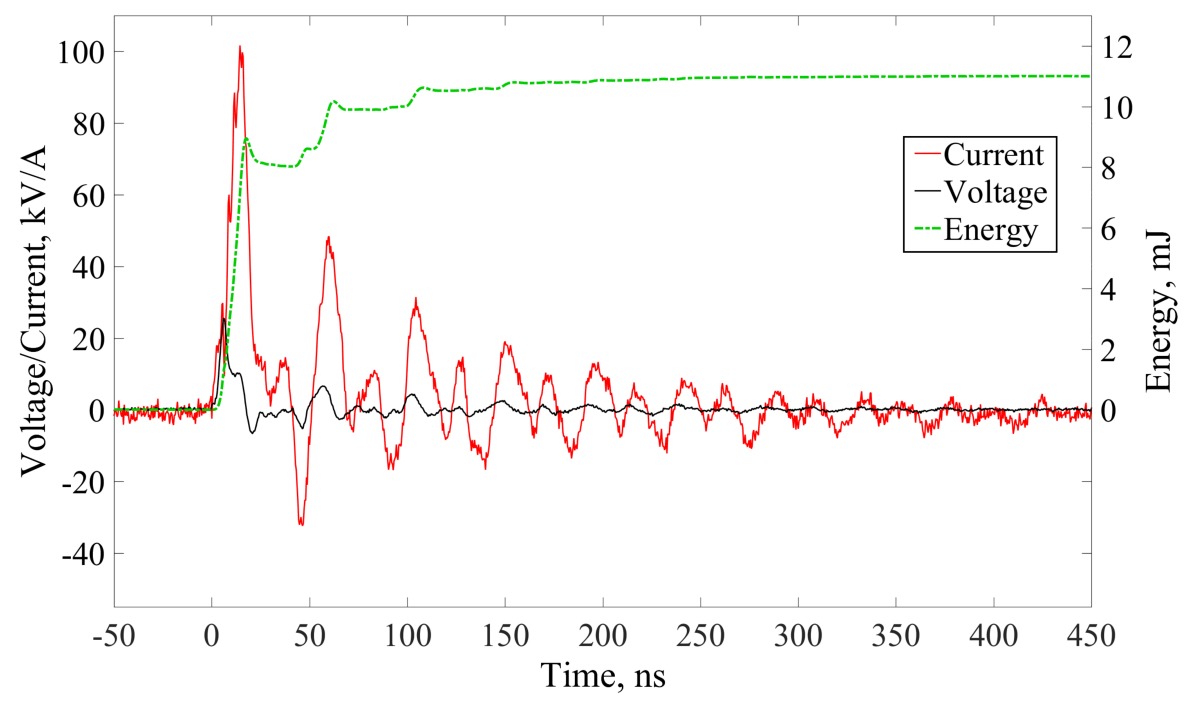

(b)

Figure 5: Example of synchronized voltage and total current signals measured during a flame acceleration event and the corresponding energy deposition (a) for a single weak NRP spark (the insert presents the energy deposition during a burst of 35 pulses for which all of the individual pulses are weak NRP sparks), and (b) for a single strong NRP spark. 


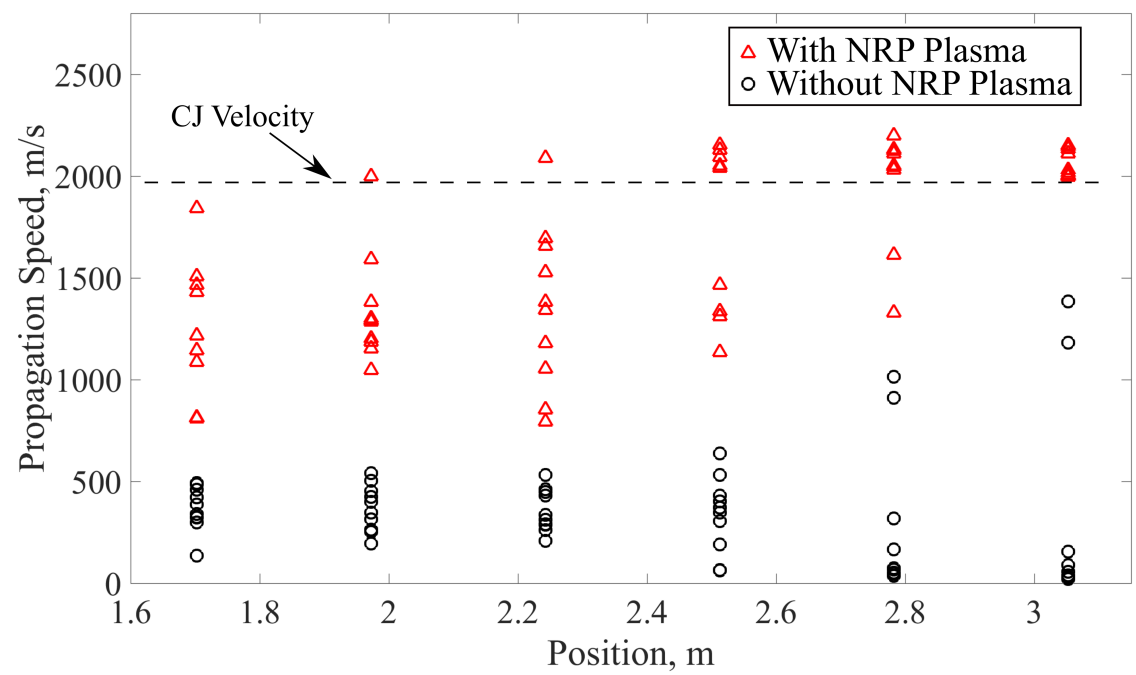

Figure 6: Development of the reaction front propagation speed obtained from time-of-flight measurements using ionization probes for cases with and without NRP plasma discharges. For the cases with plasma, a burst of 100 pulses is applied. The uncertainty in these velocity measurements is $\pm 50 \mathrm{~m} / \mathrm{s}$. 


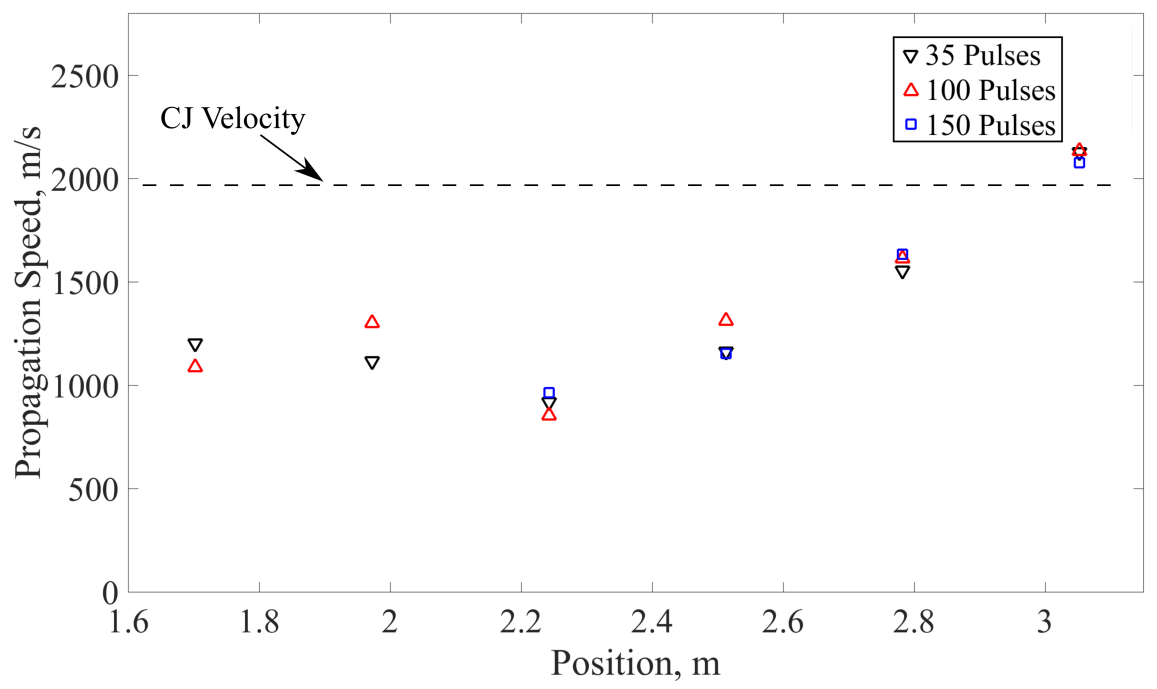

Figure 7: Development of the reaction front propagation speed obtained from time-offlight measurements using ionization probes for cases with 150 (blue), 100 (red) and 35 (black) pulses. The uncertainty in these velocity measurements is $\pm 50 \mathrm{~m} / \mathrm{s}$. 

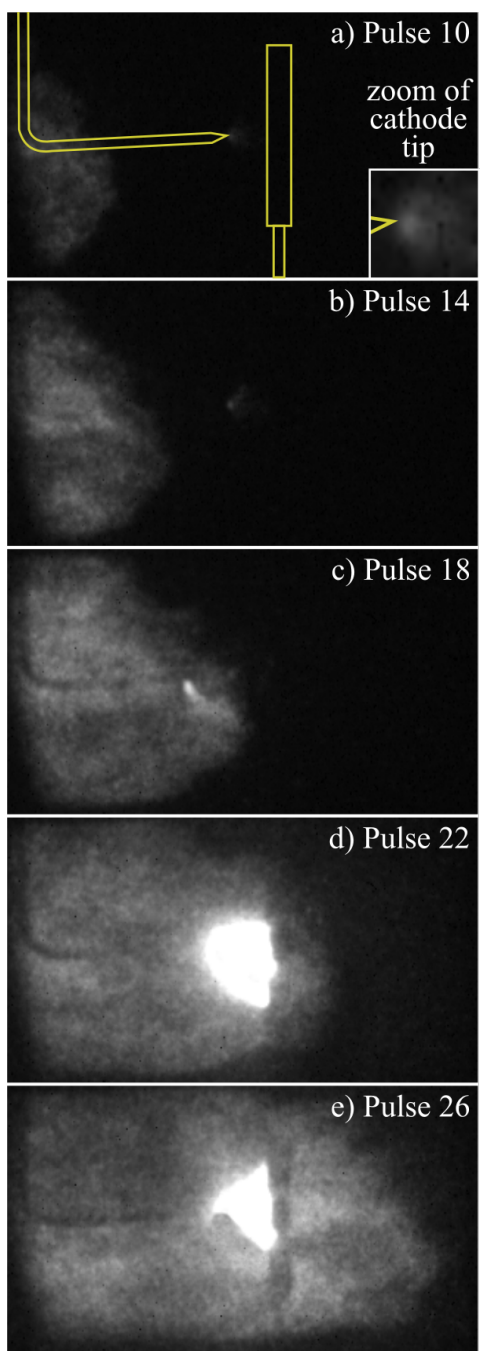

Figure 8: High-speed $\mathrm{OH}^{*}$-chemiluminescence images of the interaction between a turbulent propagating hydrogen-air flame and NRP plasma discharges. In the first image, the geometry of the electrodes is presented as well as a close-up of a weak discharge at the tip of the cathode (further enlarged and intensified). 